\title{
Mémoire et filiation dans La Nuit d'obsidienne et Retour à Satyah de François Emmanuel
}

\author{
Memory and filiation in La Nuit d'obsidienne \\ et Retour à Satyah by François Emmanuel
}

\author{
Ewa Grabowska \\ Université Adam Mickiewicz, Poznań \\ e-mail: grabowska.ewa@outlook.com
}

\begin{abstract}
François Emmanuel, renown Belgian contemporary writer and practicing psychotherapist, has so far published more than fifteen novels which are all focused on recurring and obsessive questions about memory, heritage and unknown past. The first two of his publications, Retour à Satyah (1989) and La Nuit d'obsidienne (1992) had been written during and little after his stay in Poland at the Jerzy Grotowski's Teatr Laboratorium in Wrocław. It was also the end-stage phase of the belgitude tendance, the period of intense reflexion about Belgian identity, language, literature and attitude towards the individual and national past. In the same time, an increasing interest in ancestors' lives in relation in one's identity issues started in the field of autobiographical writing (récits de filiation, hétérographie, autofiction). Although the writer never openly positioned himself about the belgitude nor has he ever written novels of autobiographical nature, his fictional works reflect the big reflexions of his time's literature. As well the thematic as the form of Emmanuel's novels echo contemporary anxieties and illustrate the functioning of memory and transmission.
\end{abstract}

Keywords: Belgium, memory, filiation, transmission, trauma, past, contemporary novel, Emmanuel

La mémoire est étrange. Les matins nous arrachent à des nuits obscures dont nous ne gardons aucune trace. Les jours succèdent aux jours et le temps dépose sans cesse cette matière d'oubli comme pour nous éloigner de nous-mêmes. 
François Emmanuel, né en 1952 en Belgique, est poète, romancier, dramaturge, membre de l'Académie Royale de Langue et de Littérature française. Il publie régulièrement et est aujourd'hui auteur reconnu d'environ vingt cinq textes poétiques, dramatiques et surtout romanesques. Parallèlement à l'écriture, il exerce le métier de psychothérapeute et psychiatre.

En 1980, il interrompit ses études en médecine pour venir en Pologne et participer pendant un an au Teatr Laboratorium de Jerzy Grotowski à Wrocław. Il a souligné à maintes reprises combien ce séjour fut pour lui significatif. Cette « expérience [...] si riche, féconde, libératrice » (Emmanuel, $2007: 30)$ sera déterminante pour la suite de sa vocation d'écrivain qui s'orientera désormais plutôt vers l'écriture romanesque. C'est lors de cette période, effectivement, qu'il rédige la version initiale de son premier texte en prose intitulé à l'époque Périple ou Archipel et qui verra le jour seulement en 1992 sous le titre de La Nuit d'obsidienne, avec une dédicace: pour ceux de Teatr Laboratorium de Wroclaw. Entre temps, il travaille sur Retour à Satyah qui, sorti en 1989, sera son premier roman publié.

Les années des premières publications d'Emmanuel correspondent à la phase finale, voire l'épuisement graduel de la belgitude, période du questionnement esthétique et identitaire en Belgique francophone (Denis, Klinkenberg, 2005 : 248). Le tournant qui s'opère à cette époque-là, animé par la belgitude et concentré autour d'elle, voit la vague néo-classique, au style canonique et à la thématique universelle, se rétrécir devant les textes de nouveau ancrés dans la réalité, réglant leurs comptes avec l'Histoire et rejouant avec le langage carnavalesque, ainsi qu'avec les genres et les esthétiques différents (Quaghebeur, $1980: 21$ ). Ainsi, parallèlement à la réforme de l'état et à l'avènement de la Belgique fédérale, voit le jour, dans la littérature belge francophone, la phase dite dialectique, qui appelle à assumer son identité plurielle, ses origines belges et surtout le caractère particulier de la langue (Denis, Klinkenberg, $2005: 226-234$ ).

C'est également le moment où l'expérience intime et l'élément biographique reviennent dans la littérature. Plusieurs écrivains, indépendamment du caractère et de l'esthétique de leur création antérieure, publient des textes qu'on qualifierait aujourd'hui d'autofictionnels: Dominique Rolin, Jacqueline Harpman, Henry Bauchau, Jean Muno dans la prose, René Kalisky, Paul Willems et Michèle Fabien dans le théâtre. Dans tous les cas, on tente d'affronter et d'incorporer dans les textes le vécu individuel et familial marqué par l'Histoire : expérience du deuil, de la disparition, des relations difficiles au sein de la famille, de l'identité trouble, de l'origine méconnue et inassumée.

Cette problématique est aussi au centre des nouvelles variations du genre autobiographique qui surgissent depuis la fin des années septante, donc parallèlement à la belgitude, surtout dans le champ littéraire français. L'autobiographie décrite et définie en 1975 par Philippe Lejeune évolue alors vers l'autofiction, terme proposé en 1977 par Serge Doubrovsky. D'ici émerge ensuite toute une famille des écritures 
de soi, désormais soumise à divers transformations et critiques: fictions biographiques (ou biographies fictives ou biofictions), égo-littérature, Roman du Je (Philippe Forest) ou encore récits de filiation (Dominique Viart, Laurent Demanze). Dans chacune de ces variantes, les chercheurs soulignent deux nouveaux aspects spécifiques par rapport aux textes à caractère autobiographique antérieurs. Il s'agit précisément du statut du sujet (Forest, $2001: 22-23$ ) et du statut de l'autre (Viart, $1999: 123,127-128)$. Le sujet, après une période de dénégation et de mise en écart, revient dans la prose fictionnelle et autobiographique, mais sous une forme différente, perturbée et modifiée surtout par les travaux de Freud et de Lacan, par « l'ère $\mathrm{du}$ soupçon » ainsi que par les expériences traumatisantes du $\mathrm{XX}^{\text {ème }}$ siècle. Dans cette période de la crise post-moderne, accompagnée de l'effondrement de repères historiques, moraux et idéologiques et de l'accélération du progrès et l'acquisition trop rapide du nouveau savoir prennent source le récit de filiation et d'autres formes d'écriture de soi.

C'est dans le contexte décrit là-dessus que le sujet commence à chercher l'identité dans le passé et questionne, dans la confusion, son histoire familiale, à travers la figure de l'autre : «Le sujet de notre temps, qui n'advient pas à ses propres désirs et s'aperçoit ne pas pouvoir même les identifier vraiment, ne peut se connaître que par le détour d'autrui » (Viart, 1999: 123, 127-128). On observe effectivement une émergence des thèmes liés à la transmission, à l'héritage et à la filiation. Ces questions qui réapparaissent dans la littérature depuis quelques décennies sont souvent marquées par une rupture ou un manque : en effet, la transmission de l'héritage se fait, aujourd'hui, dans l'absence de testament (Viart, Vercier, 2005: 18, 98), où le passage d'un savoir intergénérationnel a été rompu ou perturbé. À partir des années 1980, l'autobiographie moderne se tourne, dans son questionnement sur l'identité, vers l'Autre, et «l'écriture autobiographique est plus volontiers autofictive qu'immédiatement mimétique d'un réel avéré » (Viart, 1999: 116). Les romans - donc les textes de fiction - ont désormais « en commun de se présenter étrangement comme des romans personnels où c'est la vie d'une autre qui, en un glissement imprévu, devient l'objet essentiel de l'investigation autobiographique. [...] L'autobiographie se constitue alors en une histoire de l'autre, en une « hétérographie » $[. .$.$] et c'est dans le jeu avec cette altérité fictive qu'incertaine se déploie$ désormais l'écriture de soi » (Forest, $2001: 45$ ).

S'il est vrai que de cette thématique tend, majoritairement, à se réaliser dans les écrits à caractère autobiographique ou autofictionnel, la création de François Emmanuel prouve que ces questions occupent également des écrivains de fiction. Le romancier belge n'a jamais publié de roman qui réclame l'appartenance à l'autobiographie ou l'autofiction, la thématique de ses textes rejoint néanmoins celle qui anime les récits de filiation ou plus généralement l'écriture de soi contemporains. Rien ne nous permet de croire, dans le cas de François Emmanuel, que l'apparition si fréquente de cette problématique découle de son expérience person- 
nelle. Elle constituerait plutôt sa source d'inspiration et une pure transposition fictionnelle d'un questionnement qui ronge son époque.

À aucun moment, non plus, l'écrivain ne s'identifie explicitement à la belgitude et ses œuvres viennent une décennie après les publications principales de ce mouvement ${ }^{1}$. L'on peut toutefois tenir pour acquis qu'un certain climat intellectuel de l'époque eut l'influence sur sa création ${ }^{2}$. Celle-ci est surtout visible au plan thématique dominé par les mêmes, obsédantes questions dont la plus importante semble être celle de savoir comment vivre après un traumatisme, avec une rupture ou une absence. Ses livres touchent presque tous à la reconstruction de la vie après un traumatisme, à la recherche des origines ou à la fouille dans le passé en vue de découvrir ou forger son identité. L'on associerait aisément ses textes de fiction à la citation de Jacques Derrida sous laquelle les spécialistes de l'écriture de soi contemporaine placent souvent leurs réflexions : "Faire sa propre histoire n'est possible qu'à la condition de l'héritage » (Derrida, 1993).

Sa formation de psychanalyste n'y est probablement pas pour rien. Elle reste, au dire de l'écrivain, une activité complétement distincte de son écriture, mais il est difficile de ne pas s'apercevoir d'une certaine connivence qui s'installe entre les deux occupations et qui se manifeste, dans son œuvre, aux plans thématique et stylistique. La raison essentielle de cette approche est pourtant la valeur cardinale de la même fonction fondamentale : le récit (Piret, $2007: 5$ ).

Emmanuel réfléchit ainsi dans ses textes au caractère et au fonctionnement de la mémoire, surtout individuelle, mais il se réfère souvent aux expériences communes et universelles. Cette réflexion entraine aussi une interrogation sur la transmission de l'identité et de l'héritage ainsi que sur les rapports entre le passé et le présent. Sur ce point, les meilleurs exemples sont La leçon de chant (1996), Retour à Satyah (1989) ou encore Le sentiment du fleuve (2003). L'écrivain a d'ailleurs toujours été intéressé par les relations familiales et il en témoigne entre autres dans La Chambre voisine (2001), La Passion Savinsen (1998), Regarde la vague (2007). Implicitement, le «creux » identitaire belge et le silence sur l'Histoire, propre au Plat Pays (Laserra, 2010 : 193), trouvent leur place dans l'écriture emmanuélienne. D'autant que, sur une vingtaine de romans publiés, seulement un (Le Sentiment du fleuve, 2003) a l'action située en Belgique, identifiée comme "pays pluvieux et improbable » (Emmanuel, 2003: 15). L'écrivain dresse aussi un lien entre la thématique de ses romans et l'acte d'écrire tel quel. "L'aventure du roman n'a jamais été autre chose pour moi : un territoire intime et pourtant étranger, un lieu qui m'attire mais à l'orée duquel je ne sais rien. [...] Au seuil de l'île, le narrateur est au commencement d'une aventure quand j'étais moi, auteur, au bord de l'histoire à écrire »

\footnotetext{
${ }^{1}$ M. Quaghebeur, Balises pour l'histoire des lettres belges, 1982. P. Mertens, « L'autre Belgique», Les Nouvelles littéraires, 1976, J. Sojcher, La Belgique malgré tout, 1980.

${ }^{2} \mathrm{http}: / / \mathrm{www}$.revue-democratie.be/index.php/culture/9-culture/49-romans-francophones-de-belgiquepaysage-multiple-ouvert-a-tous-vents, consulté le 25 juin 2015.
} 
(Emmanuel, $2007: 10,12$ ). Et on se rappelle que la réflexion sur l'écriture et sur la véracité du récit accompagne souvent, chez les écrivains contemporains, celle sur la redécouverte de leur passé et par là, de leur identité.

Le rapport au passé, à ses parents ou ascendants, était la source de la plupart d'intrigues emmanuéliennes. Les personnages d'Emmanuel sont hantés par le passé « qui contient le secret de leur héritage ainsi que la compréhension de leur présent et la possibilité de leur avenir » (Parent, Schwerdtner, 2012) jusqu'à en ressentir une douleur charnelle et entreprendre un effort physique de la recherche de la mémoire : « Je ne pourrais jamais dormir, pense Aniel et il sent revenir en lui le tremblement interne, le creux, le vide, l'excavation de son corps et il frappe avec son bras bandé sur le rebord du lit. Pour chasser l'image. Il frappe jusqu'au sang » (Emmanuel, 1989 : 35). Comme il ne s'agit pas d'un passé éclairé et entièrement reconstruit, mais plutôt « d'un passé à élucider par le biais d'un récit parfois hésitant, incertain, rempli de blancs, de vides, de failles » (Parent, Schwerdtner, 2012), les récits du romancier belge, déployés autour d'une absence ou d'un manque, dépeignent les jeux entre ruptures et reprises, entre liaisons et déliaisons, entre déchirures et retours. Ils nous semblent représenter un curieux jeu et une illustration particulière de cette nouvelle convention littéraire se choisissant comme thème la recherche des origines, les relations familiales et la construction identitaire qui se fait sur la base d'un questionnement du passé. Nous voulons les analyser comme tels à l'exemple de deux premiers romans d'Emmanuel : La Nuit d'obsidienne (1992) et Retour à Satyah (1989).

\section{PRÉSENTATION DES ROMANS}

Il convient de présenter les deux textes, avant de passer à leur analyse. Aussi bien La Nuit d'obsidienne que Retour à Satyah sont de pures fictions. Les deux présentent des visions intéressantes de la narration et des récits. Leur construction particulière incite à les regarder comme illustration textuelle de la thématique abordée, soit le fonctionnement de la mémoire et la construction de l'identité. L'histoire de La Nuit d'obsidienne commence « dans le grondement des haut-parleurs d'une gare » (Emmanuel, $2002: 12$ ) où erre le narrateur, jeune médecin blasé, pour chasser l'ennui et le dégoût de sa propre vie. Il y rencontre par hasard un vieillard un peu dément qui s'avère être un ancien archéologue. Le prénommé Pierre Ansalem lui raconte sa fascination pour une île de l'Archipel Nord où il a étudié pendant des années les rites funéraires des autochtones. Au bout de quelques mois de rencontres et de conversations, le narrateur décide de partir, ce que l'archéologue espérait dès le début, sans l'y inciter ouvertement. Une fois arrivé à l'archipel, le narrateur découvre petit à petit une histoire sombre et tragique qui a fait partir l'archéologue et l'a séparé de sa fille, Ann. Le narrateur rencontre la femme et tombe amoureux d'elle, mais leur relation ne dure pas, après quoi il quitte l'île. Tout au long de son 
séjour dans l'île, le narrateur est constamment pris pour un autre, un certain Elie Maccanéis, médecin et ami des habitants, mort dans des circonstances mystérieuses.

La Nuit d'obsidienne fut très bien reçu : aussi bien les critiques que les lecteurs ont apprécié son caractère "fantastiquement onirique », fascinant et troublant, ainsi que « le travail de la langue », mélodieuse et fluide ${ }^{3}$. Le livre lui vaut aussi la première reconnaissance publique (Prix triennal de la ville de Tournai 1993). Ce récit, sans beaucoup d'intrigue au sens propre, comporte une riche symbolique et présente des explorations intérieures très développées de ses personnages. La réflexion sur l'identité et la mémoire face à un traumatisme du passé est au cœur de ce roman conçu à une époque importante, voire initiatique, pour l'écrivain, et qui semble en effet comporter déjà les grandes lignes et les balises de son œuvre à venir. Le thème existentiel, l'intemporalité universelle et les espaces symboliques - que nous allons développer par la suite - nous invitent à lire ce texte comme roman-phare de la création emmanuélienne, un livre où se dévoile « le fondement même de (sa) pensée » (Emmanuel, 2007 : 4, 11).

Retour à Satyah apparaît comme prolongement de l'optique choisie par La Nuit d'obsidienne et en tant qu'actualisation du système esquissé ci-dessus. Tout en adoptant un schéma de base pareil, le roman est davantage ancré dans la réalité, comporte des repères plus précis et fréquents.

Une fois encore, l'histoire se déploie autour d'un vide. Au cœur de Retour à Satyah se trouve l'absence de Hanna, mère du personnage principal, Aniel Mahasöhn, qui grandit entouré du non-dit de son origine. C'est seulement à l'âge de 8 ans qu'il apprend de son oncle qu'il était le fils d'Hanna, sa sœur, jeune juive morte pendant la guerre dans le ghetto de Bialystok. Aniel « craignit de poser d'autres questions [...] [et] grandit dans ce précieux silence de la phrase inachevée » (Emmanuel, 1989 : 44). Personne ne lui parle de sa mère et le sentiment de déracinement et d'aliénation s'aggrave au point d'influencer toute sa vie. Il vit entouré de nombreux membres de sa famille, mais le vide en lui est tellement grand qu'il l'empêche de vivre normalement et le pousse à aller chercher toujours plus loin l'histoire de la mère inconnue.

La thématique du roman oscille autour de quête identitaire, voyage initiatique et recherche des origines. L'on suit le cheminement et les rencontres consécutives d'Aniel cherchant tantôt les traces de sa mère, tantôt l'oubli total dans le chaos et dans les bruits du monde. L'importance de la connaissance de ses racines et de son héritage est donc au cœur de l'intrigue - comme moteur d'action - et au centre de l'intérêt d'Emmanuel. La structure du roman, beaucoup plus développée que celle de La Nuit d'obsidienne, reste en rapport étroit avec la thématique et c'est elle qui nous intéressera particulièrement comme représentation du fonctionnement de la mémoire et des relations de filiation.

\footnotetext{
${ }^{3}$ Extraits de critiques de presse.
} 


\section{TEMPS ET ESPACE}

La plus grande partie de l'histoire racontée dans La Nuit d'obsidienne a lieu sur une île d'un archipel dans le nord ; l'espace continental d'où venait le narrateur n'est aucunement déterminé. On tombe sur quelques noms des lieux comme Longhone, Saan, Semaan mais ils ne nous informent pas davantage sur la situation de l'archipel. Malgré quelques indications de dates plus ou moins précises qui parsèment çà et là le récit, le lecteur plonge vite - à l'instar du narrateur - dans une inquiétante intemporalité. L'on sait pourtant, par des indices vagues et imprécis, que l'histoire se déroule à l'époque contemporaine : l'événement déterminant pour toute l'histoire est la découverte des champs pétroliers dans la mer entourant l'île, on se déplace avec des moyens de transport modernes et on tue avec des mitrailleuses. Des précisions ou des références directes à une quelconque actualité sont pourtant absentes, on n'évoque pas de nouvelles de presse ni d'informations actuelles. Ceci renforce l'impression que l'archipel est soumis à un autre cours du - ou des - temps. L'île est, en effet, l'espace de superposition de plusieurs couches temporelles. Le temps d'après le retour où le narrateur fait son récit est le plus proche du lecteur. Le temps suspendu des insulaires dont l'histoire rapporte le narrateur - forme la seconde couche. Enfin, le temps archaïque de l'île est érigé par les propos du vieil archéologue et rappelle l'époque des rites mortuaires primitifs, ouvrant ainsi la dimension du mythe qui se déployait autour du mystère de la mort. Cette dimension, par sa présence discrète et transparente, constitue un fondement pour la pensée incluse dans le roman : le passage de la vie à la mort, autrefois entouré de sacre et de rites, était ainsi apprivoisé et assumé. L'espoir de la vie posthume et un rituel répétitif permet de garder la continuité, d'éviter la rupture et d'incorporer la mort comme élément naturel de l'existence humaine afin de pouvoir continuer à vivre. L'expérience de la mort dans le roman n'a rien de paisible, elle rompt les liens et bouleverse les êtres. Les habitants de l'île apparaissent comme bloqués dans un moment tragique du passé, ils y reviennent constamment et semblent coincés dans une histoire inassumée qui les empêche de progresser et de vivre normalement.

Cette suspension est également visible dans les descriptions de l'espace. Le paysage sévère, sauvage et pierreux couvre la vaste surface de l'île. Cette ouverture «physique » de l'espace n'a rien à voir avec la facilité d'y accéder : ni mentalement - les insulaires étant farouches et méfiants ni physiquement, car le voyage du narrateur était une démarche difficile et il a fallu recourir à l'aide d'un passeur, à des lettres clandestines, à des connaissances. C'est également un lieu inquiet et déchiré. À son arrivée, le narrateur découvre un monde partagé, une part de l'île étant occupée par les militaires protégeant la raffinerie, l'autre - sauvage et oubliée, éloignée de la civilisation habitée par les indigènes. L'enclos militaire fut quelques années auparavant la scène des luttes acharnées et sanglantes entre les indigènes et les étrangers arrivés exploiter la richesse de l'archipel. Ce lieu reste désormais sous 
garde constante de l'armée, empreint de tabou et de silence parmi les insulaires. Le lecteur et le narrateur sont conscients seulement de son existence et des rancunes qu'il éveille, mais on n'en sait rien d'autre - ni sur les gens qui y vivent ou travaillent, ni sur ce qui s'y passe actuellement. La raffinerie reste ainsi - symboliquement et réellement - un trou noir autour duquel gravitent les souvenirs et les vies des habitants de l'île, absorbant leurs pensées, bouleversant le passage du temps habituel. Son espace clos illustre le traumatisme inavoué, incompris et refoulé, autour duquel se construit, tant bien que mal, la vie d'après. L'espace ouvert du paysage infini et désert de l'île est celui où errent les insulaires suspendus dans le temps, remémorant des événements passés, attendant encore les retours impossibles. Pierre Ansalem invitait le narrateur « à [s']imprégner des lieux, [...] comme si tout était là, dans la chair blessée de l'île, sur les aplats décolorés des murs, les orbites colmatées des fenêtres. Et dispersé dans le vent, confondu à la cendre du jour, le corps partout d'Elie Macchanéis » (Emmanuel, 2002 : 53-54). L'invitation à l'observation et à la simple présence est donc là : la compréhension se fera hors du vacarme, en arrêt de la course normale de l'existence, dans un présent différent du quotidien.

Une telle description de l'île enrichit l'imaginaire du détachement propre au roman. Sur ce détachement, qui forme une déchirure, se fondent les jeux d'oppositions entre le clos et l'ouvert, la nuit et le jour, l'ici et l'ailleurs, le présent et le passé, qui mènent tous à cette opposition cruciale entre la vie et la mort. L'économie d'indicateurs spatio-temporels incite à interpréter les éléments du récit comme symboliques et à lire l'histoire dans son acception universelle. Il importe aussi de remarquer que l'île fait partie d'un archipel : l'évocation de la formation de plusieurs éléments semblables transpose la réflexion sur un plan universel où l'expérience humaine est répétitive, multipliée, récurrente, indépendamment des époques et des circonstances.

\section{EN QUÊTE}

Il y a toujours un moment décisif du départ - le premier pas dans l'aventure du passé qui passe forcément par sa dimension purement physique - il faut d'abord changer de lieu, partir, rompre - en quelque sorte - avec le présent. « Aller au plus loin pour aller au plus près, si l'on veut se connaitre, prendre un chemin qu'on ne connaît pas » (Emmanuel, $2002: 12)$ : ces mots que l'archéologue adressa au narrateur de La Nuit lors de leur première rencontre pointent dès le début la nature existentielle et intérieure des déplacements et suggèrent la dimension métaphysique de l'espace et du temps.

Une immense gare où le narrateur rencontra Pierre Ansalem devient le premier des espaces symboliques de La Nuit d'obsidienne, effectivement placé sous le signe du départ. «Je hantais comme lui ces lieux de partance et je crois que nous avions 
l'un et l'autre la même rôdeuse immobilité » (Emmanuel, 2002 : 12). Cherchant à comprendre les raisons de sa volonté de partir, le narrateur parle des attaches qui sont devenues lâches. Il évoque un sentiment de vide, de détachement, de vanité, « une sorte de trépidation vaine où chaque geste, chaque mouvement [le] renvoyait au désert d'être [...], où [il croyait] partout [se] cogner comme aux murs invisibles d'une chambre » (Emmanuel, 2002 : 11). En proie au trouble existentiel, peu engagé dans son travail, le narrateur ne trouve pas de satisfaction, n'entretient aucune relation profonde et semble de plus en plus détaché d'un monde qu'il connaît : «un monde sans ailleurs, sans jachère, sans dieu et sans monstres », tel « une boule qu'on tient entre les mains et dont on peut presque tout connaître » (Emmanuel, $2002: 12$ ). Le narrateur porte ainsi en soi une sorte de déchirure élémentaire et un détachement du monde dépouillé et plat. L'on peut y voir le reflet de la crise du monde postmoderne dans lequel naquirent les nouvelles espèces de la réflexion autobiographique (Demanze, 2014 ; Viart, 2011 : 16-17). Ce vide intérieur le poussera à partir intuitivement, à la recherche d'une expérience spirituelle imprécise, d'une essence inconnue, d'une rencontre peut-être avec ces dieux et ces monstres dont son existence était dépourvue.

Les départs sont toujours des recherches. Pour comprendre le mystère de l'île, le narrateur de La Nuit doit se laisser porter par le récit des autres et y entrer complètement, il cherche donc des traces, des morceaux, des indices. Il doit les rassembler et comprendre l'histoire qui - au premier coup d'œil - n'est pas la sienne. «Revenez au paysage encore, revenez aux ciels, aux anciens endroits de passage, aux routes qu'empruntaient les hommes... » (Emmanuel, 2002 : 54). Le processus de compréhension est ainsi placé sous le signe du retour, de la réappropriation d'un savoir jadis connu et d'une reconstruction d'une image brisée.

Les répétitions et les reprises très fréquentes renforcent l'impression d'un éternel retour. Le narrateur se voit immédiatement entraîné dans une boucle répétitive de la mémoire inapaisée des autres. L'archéologue, d'abord, ne cache pas devant le narrateur qu'il voudrait « revoir l'île avec la jeunesse de ses yeux » (Emmanuel, 2002 : 19). Le narrateur refait donc le voyage du protagoniste, plonge dans sa mémoire toujours vive, marche à tâtons dans le passé d'un autre. Elément extérieur et nouveau, il est comme un prisme à travers lequel les personnages veulent revoir le passé. Quand une habitante de l'île désire lui montrer de vieilles photographies, le narrateur a l'impression que «Jana veut qu'[il] entre dans un temps qui n'appartient qu'à elle » (Emmanuel, 2002 : 45). Ainsi, mené par des bribes de souvenirs, une parole échappée ou une photographie, il découvre que le mystère de l'île est lié à la mort tragique d'un certain Elie Macchanéis, médecin et ami des insulaires, amant de la fille de l'archéologue, Ann. Une multitude d'objets ressurgissent aussi autour du narrateur, "pris par la fièvre à fouiller dans [le] réduit ces caisses, ces tiroirs, le double fond d'une armoire qu'on a curieusement bourrée de crêpe noir ». Il y retrouve « des vieux registres comptables, des lettres, une carte de l'île, [...] des 
écailles de pierre sombre aux stries concentriques (Emmanuel, 2002 : 46). Les insulaires, en effet, laissent le narrateur entrer dans le cercle très intime de leurs mémoires et blessures, afin qu'il sache, qu'il raconte, qu'il prolonge leurs histoires.

Si les habitants de l'île l'avaient admis dans leur communauté, pourtant, c'est parce qu'il leur rappelle trop Elie pour être considéré comme étranger. Il profite de la confiance que son prédécesseur a gagnée, il refait ses chemins et reprend ses habitudes - dort dans sa chambre, soigne ses patients, aime son amante. La confusion va jusqu'à son nom : on lui demande s'il est fils ou frère d'Elie, s'il est venu continuer son œuvre. Des vieillards qu'il soigne l'appellent ouvertement «Elie » et Ann, qui deviendra son amante, soupire ce prénom aux moments de l'étreinte amoureuse. C'est ainsi qu'il se sentait attendu par des gens qu'il n'avait jamais vus. Son hôtesse, curieusement, « observe qu'elle ne [l']avait jamais imaginé si jeune » (Emmanuel, 2002 : 36). Il est obstinément perçu comme un autre, forcé à entrer dans son rôle et à remplir le vide laissé par lui pour que, grâce au voyage à rebours, l'histoire interrompue puisse se jouer jusqu'à la fin et que ses personnages sortent du rouage vicieux du récit inachevé. Ann Ansalem, par sa liaison avec le narrateur, complète symboliquement la relation tragique avec Elie, dépasse sa perte et arrive à continuer sa vie. Le départ d'Ann devient pour le narrateur amoureux d'elle son vide initial à lui et entame sa propre attente : ainsi l'histoire aboutie pour quelqu'un marque le début de la sienne, inachevée. La mémoire « emmanuélienne » est ainsi cyclique et répétitive, elle tourne en boucle, jouant obstinément la même vieille mélodie.

L'errance - seulement annoncée dans La Nuit d'obsidienne - prend de l'ampleur dans Retour à Satyah. L'histoire du roman est organisée en étapes répétitives autour des va-et-vient de son protagoniste. Aniel part, revient, reste, part de nouveau, toujours agité par la faim du savoir sur sa mère. Du Danemark en Pologne jusqu'à l'Israël, il guette et rassemble les morceaux des récits sur Hanna. Les premières histoires viennent de la bouche de sa grand-mère. Vieille et agonisante, bientôt la grand-mère ne lui raconte rien de nouveau et reste coincée sur la seule image qu'elle a - ou qu'elle veut avoir - de son enfant. «Et de l'interroger parfois, il comprit que la grand-mère ne livrerait jamais ce que fut sa mère, mère. Elle ne le savait pas, d'ailleurs, elle ne le savait plus, elle l'avait oublié » (Emmanuel, 1989 : 46). Aniel part donc pour écouter Wanda, une amie de jeunesse de sa mère, revient à la maison pour cueillir quelques mots de son oncle et se rend ensuite en Pologne pour rencontrer entre autres les sœurs Michałowskie, Samuel Mann, Abraham O. Tout au long du roman, s'enchainent des départs, des rencontres, des silences. Inquiet, toujours déchiré entre l'irrésistible envie de partir et l'attente immobile de récits sur sa mère, Aniel cherche tantôt à se réfugier dans la musique et dans la solitude qui le plongent dans l'harmonie et la simplicité, tantôt à se perdre dans la foule, dans la guerre, dans le vacarme et le chaos. Les périodes d'évasion et de recueillement rappellent le rôle de la distance et du temps dans la compréhension de soi-même, ainsi que l'importance du silence qui permet d'entendre le récit et de l'organiser en harmonie. 
Toute tentative d'un apaisement ou d'un refoulement est néanmoins temporaire et à la longue s'avère vaine. Ce mouvement onduleux - en avant, en arrière - contribue à l'image de la mémoire mouvante, de la mémoire pareille aux vagues de la mer, à laquelle les références ne manquent d'ailleurs pas tant dans Retour à Satyah que dans La Nuit d'obsidienne. Il réinstaure aussi le jeu d'oppositions et de répétitions : entre la parole et le silence, le vacarme et le calme, les départs et les retours.

L'image d'Aniel longeant le bord de la mer en Israël est frappante : la frontière étroite et floue entre l'eau et la terre, l'oubli dans le vacarme et l'enracinement dans la mémoire. Une phrase placée sur une des premières pages du roman nous en avertit d'ailleurs à l'avance : «nous errons entre terres et eaux cherchant à reconnaître l'endroit d'où leur voix s'élève encore, reliant par instants ces fragments du souvenir, ces récits toujours interrompus...» (Emmanuel, 1989 : 9). Pareillement dans La Nuit d'obsidienne, où nous retrouvons le renvoi que le professeur Ansalem adresse au narrateur partant à l'ile :

Ne posez pas de questions. [...] Laissez se refaire autour de vous l'ordonnancement que votre venue un instant aura troublé. Car sans doute il y a un ordre dont à votre arrivée vous ne pourrez saisir le sens. Il y a un silence dont vous ne comprendrez que plus tard l'enjeu. [...] Il faut entendre ce silence. Il faut le supporter (Emmanuel, $2002: 53$ ).

Le retour au passé peut apporter des réponses ou, ce qui se produit plutôt dans les univers emmanuéliens, éclairer des doutes, car il n'y a jamais d'une réponse certaine. Les voyages des protagonistes des romans portent en effet des traits d'une quête initiatique qui lui permettra de renouer les liens avec sa vie. Dans La Nuit d'obsidienne, le narrateur quasi-aveugle est guidé par les sages de l'archipel: le livre comporte trois parties dont chacune porte le nom d'une femme qui le menait, l'inspirait, l'instruisait enfin pendant le séjour et ainsi chaque partie apparaît comme une étape suivante de l'initiation. Le narrateur de Retour à Satyah se fie également aux paroles des autres qu'il cueille un peu partout et qui l'approchent de la vérité. Cette vérité qu'ils trouvent est souvent douteuse, mais ils peuvent accéder à une certaine connaissance universelle ou à celle d'eux-mêmes. Ce qui importe finalement dans les récits emmanuéliens, c'est le chemin et l'évolution de celui qui cherche la mémoire et la parole plutôt qu'une vérité absolue des faits qui n'existe guère : un fait, un fragment du récit est seulement vrai pour celui qui le raconte et au seul moment de le faire. Ce qui semble réel, c'est l'évolution du protagoniste à force de découvrir le passé et de travailler la mémoire. Il se peut pourtant aussi que la seule vérité soit l'acte de raconter et d'écouter.

Chaque aventure dans le passé change celui qui l'entreprend. Les narrateurs s'engagent dans les histoires qui deviennent les siennes et s'ouvrent, inconsciemment, à leur propre quête. Le narrateur de La Nuit avoue à la fin avoir été vidé de son malaise existentiel, avoir pris «d'immenses réserves de souffle » pour pouvoir enfin comprendre « la part de défi splendide liée au dessein de guérir et de soigner » 
(Emmanuel, 2002 : 192). En même temps, épris d'Ann sans réciprocité, il reste suspendu et accroché au souvenir de la femme aimée et disparue de sa vie. Le récit que le narrateur nous fait d'Aniel après sa mort témoigne de son besoin à lui de revenir en arrière pour comprendre sa perte. Les récits des narrateurs, dans La Nuit d'obsidienne et dans Retour à Satyah, prouvent qu'ils doivent à leur tour puiser dans le passé pour compenser les disparitions. La suspension et l'errance s'avèrent contagieuses.

Le mystère des origines plonge des êtres dans l'errance éternelle à leur recherche. Le traumatisme inassumé les met dans une interminable suspension dans l'attente d'un retour ou d'une réponse, symbolisés par le cortège de lieux et de visages de Retour à Satyah et par l'espace immuable des îles dans La Nuit d'obsidienne.

\section{FILIATIONS}

Le traumatisme dans les deux romans revient, en fin de compte, au seul mystère originel de la mort. L'histoire racontée est donc celle de la confrontation de la disparition et du deuil. Dans cette expérience de la rupture, l'instinct pousse les gens à chercher des liaisons, à recoudre les déchirures. Ces tentatives vont dans plusieurs sens. Ils cherchent des ascendants, pour s'ancrer dans le passé et l'apprivoiser, et des descendances : pour transmettre, pour passer le relais, pour exister un temps encore dans la mémoire de l'autre. Ainsi, La Nuit d'obsidienne, ce « récit d'une mort et d'une renaissance » peut se lire aussi comme « un texte visionnaire sur le thème de la transmission cachée » (Emmanuel, $2002: 4^{\mathrm{e}}$ de couverture). Les relations de filiation qui se nouent chez Emmanuel ne sont pas uniquement celles de sang ou de communauté de nom, mais les liens génétiques jouent eux aussi un rôle important.

Quand le narrateur de La Nuit d'obsidienne cherche à comprendre les raisons de son malaise existentiel et de son départ subit, il avoue que sa «mère a toujours été très douce » et qu'il a « trop reçu et trop vite » (Emmanuel, 2002 : 12). Au lieu de mentionner le père par la suite, il passe à la description de la rencontre fortuite de l'archéologue. Son évocation fait ainsi immédiatement penser à la figure paternelle et incite à considérer leur relation dans cette lumière. D'autant plus qu'à l'époque où commençait l'amitié avec Pierre Ansalem, "personne n'était là pour le secourir» et le narrateur dut s'occuper de lui «comme d'un vieux père». C'est alors qu'il apprit «qu'il avait une fille mais que tout contact avec elle avait été rompu depuis son départ de l'ile » (Emmanuel, $2002: 21$ ). Il le crut très affecté par cette absence, même si le vieil homme restait discret sur le sujet. Visiter l'île, c'est accéder à l'espace où vécut le vieux archéologue qui eut pris pour le narrateur la place du père, l'espace de son passé (Emmanuel, 2007: 9). Le narrateur reçut, au début de son séjour à Semaan, une lettre de la fille de l'archéologue où elle précisait qu'ils avaient laissé vieillir entre eux, son père et elle, un très long silence qui concernait l'île à laquelle étaient liés autant son destin que le sien. « Mon père ne croyait pas au hasard [...]. Il aimait parler des intimes filiations qui se nouent entre 
les êtres - ajouta-t-elle. Je ne vous connais pas et je suis surprise de m'ouvrir à vous sans que nous nous soyons jamais rencontrés » (Emmanuel, $2002: 60$ ). Ann cherche à s'approcher de son père décédé en essayant de se lier d'une filiation intime avec un homme auquel son père faisait confiance et en suivant sa règle de ne pas croire au hasard. Le narrateur retrouve en elle une amante éphémère et fugitive qui éveilla en lui des passions oubliées, à la différence d'une «autre femme [qui 1]'ayant aimé s'était flétrie dans [ses] bras » (Emmanuel, $2002: 13$ ).

La figure de Jana, une vieille villageoise qui l'accueillit dans sa maison, rappelle vaguement - par ses soins, sa délicatesse et sa chaleur - la figure maternelle lointaine. C'est au moment où le narrateur apprend sa mort et voit son corps allongé sur la table que lui reviennent des sentiments imprécis de son enfance et qu'on perçoit un bout de son mystère familiale, d'un vide refoulé à lui:

Au travers des mailles du tissu, je voyais transparaitre la robe fleurie de Jana, me sentant aussitôt emporté par un sentiment intense et double : les couleurs de la robe explosaient d'allégresse tandis qu'un sanglot noir gonflait à l'intérieur de moi, cherchait à éclater à l'air comme un chagrin très ancien, enfermé depuis des années. [...] Il me semblait que j'avais à portée de moi la fin de la phrase mais que le chagrin en voilait la lumière, un chagrin de moi-même, de ma vie oubliée, de l'enfance comme un grand manteau tiède qui m'enveloppait le cœur (Emmanuel, $2002: 106)$.

Le narrateur venu dans l'île forme une trame qui relie les insulaires à leur passé, mais lui aussi, il y retrouve les liens dont le manque il semble ne s'être même pas rendu compte. Pierre Ansalem comme père, Jana en tant que mère, Singa - enfant et Ann - amante, il se construit sur l'île une nouvelle configuration familiale qui permettra autant à lui qu'aux insulaires de combler les vides et clore les histoires inachevées, afin de revivre de nouveau ou de mourir en paix.

L'errance d'Aniel de Retour à Satyah était faire en vue de retrouver la filiation perdue, une trace des origines. $\mathrm{Si}$, au bout de sa quête, il ne trouve pas de réponse unique à sa propre existence, il accède néanmoins à une vérité générale, à une expérience universelle de la guerre et de la mort. Il y avait de nombreuses Hanna remportées par les vents de l'Histoire, chacune avec sa petite histoire inconnue, et il y avait plusieurs bébés morts et plusieurs orphelins anonymes. Il aurait pu mourir, elle aurait pu vivre. L'ombre d'Hanna, celle qui ne fut pas sa mère, flotte tout de même sur chaque rencontre, apparaît dans chaque conversation : elle est le point commun qui lie Aniel à des étrangers qui l'accueillent comme s'il était de la famille, heureux de pouvoir partager leur fragment de l'histoire. N'ayant pas trouvé le nom ni le sort de sa vraie mère, il sent pourtant, à la fin du récit, qu'il porte en lui l'image de la mère, une empreinte d'un amour universel, une présence ou une conscience acquise par l'effort de la recherche, par le travail de reconstruction de la vie d'une autre.

Il a réalisé aussi que sa grand-mère, n'ayant pas trouvé de bébé sous l'adresse indiquée, mais sachant qu'Hanna fut enceinte, s'était accrochée à cette idée et 
" elle cherchait comme folle un enfant, un enfant de cet âge. Pour accoler le nom » (Emmanuel, 1989 : 87). Aniel vint à comprendre que la grand-mère « avait laissé sa fille à l'état d'enfance dont lui, Aniel, fut la figure miraculeusement survivante. Un enfant chassant l'autre, renaissant de l'autre et le perpétuant, annulant d'un seul coup cette question folle : qu'es-tu devenue, chair de ma chair, mon amour, après l'enfance, qu'es-tu devenue ?» (Emmanuel, 1989: 48). La vieille femme cherchait à prolonger l'histoire de sa fille morte, assurer une descendance pour sa mémoire, de même qu'Aniel voulait connaitre son ascendance. Il sent ainsi en lui l'esprit du bébé mort avec Hanna dans des marais polonais, dont il a joué le rôle toute sa vie. On tient énormément, dans le roman, à assurer - par tous les moyens possibles - une quelconque transmission, un prolongement de sa lignée, de son nom, de sa mémoire. Les fantômes toujours présents réapparaissent donc dans ce roman d'Emmanuel, et font réapparaître aussi le jeu de répétitions et de reprises.

Entre le temps des souvenirs de la période de guerre et le temps des confidences d'Aniel au narrateur, encore un fil est tissé dans la matière du récit, un épisode de sa vie est mis en avant, celui de la rencontre et la relation d'Aniel avec une Palestinienne, jeune mère, victime de guerre qu'il rencontra et qu'il suivait pendant son errance en Terre Sainte. C'est une histoire qu'on dirait superposée et parallèle au reste du texte. Vagabondant avec un nouveau-né, ne parlant pas la langue d'Aniel ni celle de ses oppresseurs, la femme est une véritable transposition contemporaine de l'histoire d'Hanna et d'autres femmes de toutes les guerres. C'est en elle qu'Aniel trouva une mère. Quand il tombe malade, « la femme le couvre, la sœur, l'intruse, l'infinie familière, l'étrangère au corps doux et froid comme la mort. Elle parle la langue de toutes les mères, elle souffle sur la douleur [...]. Elle disparaît. [...] Il voudrait la retenir. Il pleurerait qu'elle le quitte [...], il pleurerait comme un enfant » (Emmanuel, 1989 : 58-59). La femme n'avait pas de nom et Aniel disait : elle, la Palestinienne. «Il eût tout donné pour savoir un nom. Parfois, sans transition, il parlait de Hanna » (Emmanuel, 1989 : 104).

Les filiations renaissent entre des personnes inconnues et dans des temps différents. Les époques s'enchainent, les récits s'entremêlent, les perspectives se superposent, mais au fond, l'histoire est toujours la même. La Palestinienne anonyme peut être Hanna perdue dans le temps, un enfant trouvé par hasard - que ce soit à Bialystok ou à Jérusalem - peut porter le nom d'une famille danoise et partir à la recherche d'une « mère à nous tous » (Emmanuel, $1989: 56)$.

\section{RÉCITS}

Le noyau des histoires entremêlées, cet événement fondamental qui bascule les vies et qui force les protagonistes à toujours chercher à le comprendre, n'est jamais dévoilé. L'on n'apprendra pas - ni lecteur, ni narrateur, ni protagoniste - comment 
et quand mourut Hanna ni qui était la vraie mère d'Aniel, comment et pourquoi disparut Elie ni ce qui s'est passé sur l'île. " A l'occultation de l'événement, le récit s'offre ainsi comme l'unique alternative » (Piret, 2007 : 5). Les événements valent moins que la parole et s'ils arrivent, c'est justement à la recherche de cette dernière.

La particularité des romans analysés réside sans doute dans le fait que le narrateur rapporte un récit d'un autre plutôt que son histoire propre. Les deux textes sont narrés à la première personne. Le je est un narrateur homodiégétique, mais ne peut pas être identifié à l'auteur. Le narrateur est toujours proche des événements et il en fait souvent partie. Il n'est pourtant jamais le personnage central ni de son récit, ni des événements racontés. Il est juste un élément de l'histoire d'un autre et il se trouve qu'il nous la raconte. Regardant constamment cet autre et le suivant dans son cheminement, les narrateurs de La Nuit et de Retour se mettent volontiers à l'écart jusqu'à paraître, par moments, transparents. Dans les deux cas, l'histoire est racontée depuis le présent, une fois les événements terminés. Le narrateur connaît donc, au moment de raconter, le déroulement presque entier des événements. Son récit est le fruit d'une enquête et il se présente souvent sous une forme inachevée.

La vision rétrospective est caractéristique pour les récits des origines, les biographies et les autobiographies. Elle nécessite pourtant un certain travail de la mémoire, un effort de remémoration, une recherche de paroles et un tri de souvenirs. Les métiers des personnages principaux ne semblent pas, sur ce point, être choisis de manière fortuite. Dans La Nuit d'obsidienne le personnage central est archéologue, profession par excellence et plus que les autres associée au passé, à son étude, au rassemblement de morceaux fragmentaires et à la reconstruction à leur base des événements refoulés. L'archéologue en question étudiait des rites mortuaires - un trait qui nous en dit long sur la nature d'événements qui seront reconstitués dans les histoires emmanuéliennes. Le narrateur, quant à lui, est médecin et en effet, son séjour à l'île chasse quelques fantômes du passé et guérit des langueurs inapaisables.

Le statut de l'Autre, encore un trait particulier des questionnements de la littérature contemporaine, se manifeste chez Emmanuel dans plusieurs relations qu'il dépeint et qui concernent non seulement l'ascendance, mais aussi la descendance. Les protagonistes, d'abord, qui doivent résoudre le mystère de vie et de mort d'un autre de leur passé pour progresser dans leur propre vie. Pétrifiés dans la douleur ou dans l'attente, ils ont besoin d'un élément déclencheur. Dans La Nuit d'obsidienne, le vieil archéologue, conscient d'être au bout de ses jours, semble pressentir que l'arrivée à l'île d'un élément étranger - un jeune homme ayant peu de liens avec sa propre existence - allait bouleverser le marasme dans lequel plongeaient depuis des années les insulaires et mettre en marche les mécanismes du deuil et de la mémoire. «J'ai pensé un instant que rien ne s'était passé »- murmura l'hôtesse du narrateur lors qu'elle l'accueillit dans sa maison. En lui donnant la clé de l'atelier, gardé intact depuis la disparition du dernier médecin du village, elle ajouta "c'est à vous » (Emmanuel, 2002 : 39). La réouverture du passé et le réveil de ses fantômes sont 
rendus très clairs par ce geste symbolique. Mais les personnages ont également besoin du regard, de l'oreille et surtout de la plume d'un autre pour rendre compte de leur cheminement, pour véhiculer le récit et transmettre l'histoire récemment découverte. Le rôle du narrateur dans le cheminement des protagonistes et dans la transmission de leurs histoires confirme ainsi l'importance de l'altérité dans la réflexion sur l'identité et la mémoire.

Il est notoire aussi qu'une toile couvre également les figures des narrateurs : on ne sait rien ou presque de leurs vies antérieures à la rencontre du protagoniste, pas plus sur leurs motivations ni leurs traumas à eux. On aurait même l'impression que, simplement, ils ne sont pas encore partis à leur recherche, qu'ils ne les avaient pas encore assumées. Dans La Nuit d'obsidienne, Ann quitte l'île, dans Retour à Satyah, Aniel meurt. Les narrateurs, ayant rapporté leurs histoires, restes seuls, maintenant avec leur passé à eux à retravailler. Les fins des livres nous laissent flotter une suggestion, un sentiment d'être au seuil d'une nouvelle histoire qui commence cette fois-ci pour le narrateur.

La chronologie n'organise plus vraiment le récit qui reflète plutôt les étapes de la lente prise de conscience, de la découverte progressive des couches du passé. Vers la fin du livre, le temps de l'histoire rejoint le temps du récit, légèrement postérieur au temps de l'histoire, après le retour du narrateur de l'île, est la perspective selon laquelle le narrateur raconte son voyage et son séjour. Il est à ce moment-là capable d'une vue d'ensemble, mais non pas d'une compréhension totale de ce qui s'est passé. Le récit du narrateur de La Nuit d'obsidienne est conçu sur la base d'un journal de voyage où il a jeté « quelques mots sur lesquels [il voulait] revenir plus tard » car, dans un "jalonnement dérisoire dans ce pays où le temps ne comptait plus, il n'y [avait] que les dates qui [étaient] certaines ». " Aussi fragmentaires fussentelles, ces notes sont le présent de mon voyage, des traces ou des preuves, même illisibles ou séparées de moi par ma propre amnésie» (Emmanuel, 2002: 22). Le récit qui nous parviendra, on en est tout de suite averti, sera imparfait et déformé par l'écoulement du temps et la faiblesse de la mémoire.

Le narrateur de Retour à Satyah dont l'on ne connaît même pas le prénom restera transparent et effacé tout au long du roman. Il raconte l'histoire d'Aniel dont il était lui aussi un élément significatif, mais il ne cherche pas à souligner sa présence. S'il s'abstient de porter des jugements ou de commenter quoi que ce soit, c'est lui qui visualise les scènes qu'il n'a pas vues et qu'Aniel ne lui a pas racontées en détails, il essaie d'imaginer les pensées et les émotions des personnes inconnues dont il transmet le récit. Patient interprète de silences, de musiques et de départs, il a entendu le récit final d'Aniel, au moment de narrer il connaît donc l'image presque complète, mais il ne la livre pas au lecteur : il la raconte en respectant la chronologie de la prise de conscience, et pas celle des événements. Il revient ainsi au lecteur de reconstituer le temps décomposé, déposé, fragmentaire; de retrouver le nœud où se nouent les fils temporels du récit, à l'instar du travail de reconstruction 
qu'a fait d'abord Aniel et puis, le narrateur. Ceci n'est pas une tâche facile. Les bribes d'histoires sautent d'une période à une autre, sans marques d'ordre ni d'indices qui permettraient de les situer dans la mosaïque d'événements. À une façon légèrement cinématographique, le narrateur enchaîne des images de différentes époques, entrepose des cadres, change de perspective, entremêle des voix.

Le chaos apparent et l'incompréhension à la première approche apprennent vite au narrateur - et au lecteur - à prendre de la distance et à faire l'effort pour coller les fragments selon sa propre intuition. Dans le cas de Retour à Satyah, la distance permettra ensuite non seulement de percevoir la grande image, mais aussi de se rendre compte par combien de lentilles doit passer l'image originelle de Hanna avant d'arriver d'abord à Aniel, puis au narrateur, jusqu'au lecteur, chacun y ajoutant et en dissimulant quelque chose. Au bout du voyage - et de la lecture - l'image de Hanna est effectivement celle d'une vieille photographie pointillée sur laquelle on la « voit » pour la première fois, un collage composé des fragments des souvenirs de différentes personnes, souvenirs qu'on sait détournés par le temps, choisis, partiaux, fades. Il s'opère alors un véritable retour au point de départ. Aniel n'acquiert pas la connaissance plus profonde de celle qu'il croit sa mère, ce qu'il apprend, ce sont plutôt les visions déformées par les années écoulées qu'ont gardées d'elle ses amis et ses connaissances de passage. La mémoire revient ainsi à un choix - ce qu'on veut retenir, ce à quoi on veut croire, ce qu'on décide encore de transmettre.

Dans l'univers des romans emmanuéliens, les aventures dans le passé - de soi-même ou des autres - apportent rarement une réponse unique et certaine. Comme si, chez Emmanuel, on devait forcément découvrir ce à quoi on ne s'attendait pas, ce qu'on n'avait pas cherché. Le narrateur de La Nuit voulait fuir l'ennui de son existence et trouva des gens suspendus comme lui, sans réponse aux questions émergeant du passé. Le protagoniste de Retour à Satyah, lui, à travers cette quête découvre qu'Hanna dont il cherchait désespérément l'image, n'était pas sa vraie mère : celle-ci serait une juive inconnue dont la seule image reste celle d'un corps informe dans un coin du ghetto. Une sorte de méfiance que le lecteur a déjà apprise à l'égard de la possibilité d'une véritable image, se déploie alors sur la valeur du récit tel quel. L'histoire racontée cesse d'être celle d'une victime de guerre et devient un récit du récit, de sa construction et de ses variantes : reconstruction et déconstruction. L'avancement du récit en même temps le décompose, bouscule le point du départ et déconstruit le savoir initial qui avait initialement poussé à la quête. Au cours du récit on apprend à s'en méfier. Mais la transmission doit se faire coûte que coûte et il est important de souligner qu'Aniel cherche non seulement des personnes de qui il pourrait cueillir des bribes de l'histoire. Il a également besoin de quelqu'un à qui raconter ce qu'il a découvert, qui transmettra plus loin son récit. Le récit, combien difficile soit-il, doit arriver, la parole - parfois trompeuse advient pour éclairer et apprendre. Le récit est pourtant modeste et la parole limitée, car elle empêche parfois l'accès à la vérité : c'est seulement après la mort de la 
grand-mère, en guise d'exemple, qu'Aniel découvre les traces de la «vraie » histoire. Vives, les personnes gardent le pouvoir de la parole, la détiennent, la forment et la manipulent. La transmission se fait ainsi davantage par la musique, le chant, les notes, les photographies, les impressions. Le silence est nécessaire pour entendre. Et à l'instar de la musique, les pauses et les silences sont aussi valables et essentiels pour la formation et la structuration du récit.

\section{CONCLUSION}

S'organisant autour d'un vide ou d'une rupture, les récits emmanuéliens développent la problématique moderne de la filiation et de l'héritage sur un mode fictionnel, sans se soustraire à des tendances observables dans le récit de filiation et les écritures autobiographiques et autofictionnelles contemporaines.

L'œuvre de François Emmanuel est animée par une réflexion constante sur la fonction du récit. Bien qu'Emmanuel souligne que le métier de psychothérapeute reste séparé de son activité littéraire, il est difficile de ne pas faire le lien entre la valeur du récit dans ses livres et le récit de vie à l'expression duquel aspirent les patients. L'accent est mis sur les nuances, sur la nécessité de narrer, de compléter son histoire, tout selon l'approche psychanalyste.

A la recherche des origines, les personnages retrouvent la parole, d'abord celle des autres, puis la leur, pour la confier ensuite au narrateur du roman qui nous la livre à nous, lecteurs. La mémoire selon Emmanuel n'est pas une langue, mais justement la parole, le langage, l'actualisation individuelle. Et la parole dans l'univers emmanuélien est pourtant très ambivalente, tantôt libératrice, tantôt impuissante voire nuisible. 


\section{BIBLIOGRAPHIE}

Demanze, L. (2012). «Le récit de filiation aujourd'hui », in Ecritures contemporaines. Atelier de recherche sur la littérature contemporaine, [en ligne] URL: http://ecrit-cont.ens-lyon.fr/ spip.php?rubrique39, consulté le 23 juin 2015.

Denis, B., Klinkenberg, J.-M. (2005). La littérature belge. Précis d'historie sociale, Bruxelles : Espace Nord.

Derrida, J. (1993). Spectres de Marx, Paris : Galilée.

Emmanuel, F. (1989). Retour à Satyah, Aix-en-Provence : Alinéa.

- (2002). La Nuit d'obsidienne, Bruxelles : Labor.

- (2003). Le sentiment du fleuve, Paris : Stock.

- (2007). Les voix et les ombres, Carnières-Morlanwelz : Lansman.

Forest, Ph. (2001). Le roman, le je, Nantes : Editions Pleins Feux.

Laserra, A. (2010). "Pour une littérature belge francophone: quelques jalons », in A. Laserra (dir.), Album Belgique, P.I.E. Bruxelles : Peter Lang.

Parent, A.-M., Schwerdtner, K. (2012). «Présentation», in temps zéro, 5 [en ligne]. URL: http://tempszero.contemporain.info/document899, consulté le 13 février 2015.

Piret, P. (2007). « Préface », in Les voix et les ombres, Carnières-Morlanwelz : Lansman.

Quaghebeur, M. (1980). «Au pays d'impossible identité », dans: Lettres belges de langue française, Liège : Europalia 80 Belgique.

Viart, D. (1999). « Filiations littéraires », Écritures contemporaines 2. États du roman contemporain, Actes du colloque de Calaceite, 6-13 juillet 1996, textes réunis par J. Baetens, D. Viart, Lettres Modernes Minard : Paris-Caen.

Viart, D. (2011). "Interroger l'histoire », in Roman français contemporain, TDC, 1007 [en ligne], URL : https://www.reseau-canope.fr/tdc/tous-les-numeros/le-roman-francais-contem porain.html, consulté le 2 juillet 2015. 
